\title{
Physical parameters of helium-rich subdwarf B stars from medium resolution optical spectroscopy ${ }^{\star}$
}

\author{
A. Ahmad and C. S. Jeffery \\ Armagh Observatory, College Hill, Armagh BT61 9DG, N. Ireland, UK \\ Received 24 December 2002 / Accepted 13 February 2003

\begin{abstract}
Most subdwarf B (sdB) stars have spectra that show helium (He) abundances depleted to a lesser or greater extent. This has been attributed to diffusion and gravitational settling. However a small but significant number of stars with similar temperatures and gravities show relatively strong helium spectra. The question is how these helium-rich sdB (He-sdB) stars are related to their He-deficient counterparts. We present physical parameters for sample of He-sdB stars using line-blanketed LTE models. From the analysis we show that the He-sdB stars are quite distinct from the He-poor sdB. We also explore the idea of a possible link between some extreme helium (EHe) stars and He-rich subdwarf O (He-sdO) stars.
\end{abstract}

Key words. stars: chemically peculiar - stars: early-type - subdwarfs - stars: atmospheres - stars: fundamental parameters

\section{Introduction}

The vast numbers of faint blue stars in our own Galaxy (Green et al. 1986) and in giant elliptical galaxies (Brown et al. 1997) are dominated by a population of subluminous B stars. These so-called subdwarf B ( $\mathrm{sdB}$ ) stars are core-helium burning stars of $0.5 M_{\odot}$ with a thin hydrogen envelope. Their surfaces are predominantly weak in helium as a consequence of elemental separation of elements in these high-gravity radiative atmospheres (Heber 1986 and references therein).

A very small number of hot subdwarfs identified in the Palomar-Green (PG) survey (Green et al. 1986), have spectra dominated by neutral helium lines, weak or absent Balmer lines and, in some cases, lines due to ionized helium. Labelled sdOD in the PG survey, investigators have variously referred to these helium-rich subdwarf B stars as He-sdB (Moehler et al. 1990a), sdB4 (Drilling 1996) and sdB:He4 stars (Jeffery et al. 1997). Here we use the term He-sdB stars to refer to the group as a whole. A list of 48 known He-sdB stars was given by Jeffery et al. (1996). Additional objects of similar spectral type have since been identified in the Hamburg-Schmidt, Edinburgh-Cape and the Sloan Digital Sky surveys (Harris 2002, private communication). More precise classifications are being prepared by Drilling et al. (2003); these characterise $\mathrm{He} / \mathrm{H}$ line ratios by an index between 0 and 40 . Here, we consider stars with an $\mathrm{He} / \mathrm{H}$ index $\geq 20$ to be He-sdB stars.

Send offprint requests to: A. Ahmad, e-mail: amir@star.arm.ac.uk

* Based on observations made with the William Herschel and Isaac Newton Telescopes operated on the island of La Palma by the Isaac Newton Group in the Spanish Observatorio del Roque de los Muchachos of the Instituto de Astrofísica de Canarias.
With strong HeI absorption lines, He-sdB stars would appear as a group to have effective temperatures ( $\left.T_{\text {eff }}\right)$ similar to normal sdB stars. To date, few analyses of single He-sdB stars have been published (Heber et al. 1988; Moehler et al. 1990a; Schulz et al. 1991; Moehler et al. 1997) and consequently little is known about their basic properties, including $T_{\text {eff }}, \log g$ and surface composition. PG 1544+488 and JL87 have been treated as prototypes in the literature for the He-sdB stars. The surface gravity of PG $1544+488(\log g=5.1)$ is lower than that of normal $\mathrm{sdB}$ stars by $\sim 1$ dex and it is extremely helium-rich $\left(n_{\mathrm{He}} \leq 0.99\right)$. JL87 also has a similar surface gravity $(\log g=5.2)$ and although it is helium-rich $\left(n_{\mathrm{He}} \sim 0.17\right)$ compared to normal sdB stars, this helium abundance is not typical of other "He-sdB" stars.

Whilst He-sdB stars are rare (there are 28 sdOD stars out of $684 \mathrm{sdB}$ stars in the PG survey), it is intriguing that they are found both in the field and in globular clusters. Single examples have been found in both M15 and $\omega$ Cen (Moehler et al. 1997, 2002).

In order to discover why the He-sdB stars are so heliumrich or, in other words, to explore their evolutionary origin, we have set out to explore their atmospheric parameters systematically. Over a series of observing campaigns we have acquired a number of spectra of He-sdB stars. While it is evident that the He-sdB classification for some of these is mistaken, the remaining sample provides the first systematic study of these objects.

\section{Observations and data reduction}

The primary observations of He-sdB stars were carried out at the William Herschel Telescope (WHT: $4.2 \mathrm{~m}$ ) in 1996 May 
using the ISIS double-beam spectrograph. Spectra were also obtained at the WHT with the Utrecht Echelle Spectrograph (UES) in 1995 May. For the UES 1995 data the resolution of the final spectra had to be degraded from the nominal instrumental resolution to the figure given in Table 1 due to low instrumental throughput.

Additional spectra have been obtained with the Isaac Newton Telescope (INT: $2.5 \mathrm{~m}$ ) using both $25 \mathrm{~cm}$ (RGO25) and $50 \mathrm{~cm}$ (RGO50) cameras of the Royal Greenwich Observatory spectrograph. Spectra were obtained over a variety of wavelength ranges between 3900 and $5000 \AA$, depending on the spectrograph, camera and detector size. The instrumental resolution range was $R \sim 2500-5700$. The stellar spectra were bias subtracted, flat-fielded and sky subtracted and wavelength calibrated using standard procedures. All spectra were normalized by defining a smooth polynomial continuum from sections of local continuum. Care was taken to avoid the wings of broad absorption lines as these are used for determining $\log g$.

The intended sample of the 1996 WHT/ISIS observations comprised as many He-sdB stars from Jeffery et al. (1996) as could be observed. The original sample was quite small. It has been expanded by observing other He-sdB stars, mostly from the same list, as opportunity arose. Of necessity, these principally comprise the brightest northern He-sdB stars.

We note that the following stars have been referred to with different identifiers in previous papers: PG 0902+057 - previously labelled 0902+058, PG 1554+408 - previously $1554+407$, PG $1600+171$ - previously $1601+171$, PG $1615+413$ - previously $1616+413$, PG $2321+214$ - previously $2322+214$ (all Jeffery et al. 1996), and PG 1544+488 previously 1544+487 (Heber et al. 1988).

Radial velocity shifts were calculated by cross-correlation between the observed spectra and approximate theoretical models described below (cf. Aznar Cuadrado \& Jeffery 2002b). The observed spectra were then corrected to the laboratory rest frame by applying the measured radial velocity shifts. The resulting spectra are shown in Fig. 1. By definition, it may be seen that the blue spectra are dominated by strong Her absorption lines. Where Balmer lines may be apparent, the presence of the intervening members of the HeII Paschen sequence may be used to determine where these are dominated by the corresponding HeII transition. We note that strong Cir lines are also present in most of these spectra.

\section{Spectral analysis}

\subsection{Model grids}

Two theoretical model atmosphere grids of high-resolution spectra were calculated. The model spectra used in the analysis were computed with line blanketed plane-parallel LTE codes STERne and sPectrum (Jeffery et al. 2001a). The first grid was used for extremely helium rich stars and was calculated on a three-dimensional rectangular grid defined by $T_{\text {eff }}=25000(5000) 40000 \mathrm{~K}, \log g=4.0(0.5) 6.0$ and $n_{\mathrm{He}}=$ $0.9480,0.9880$ and 0.9970 . A second grid which was used for stars with lesser helium abundances was calculated on
Table 1. Spectroscopic observations of He-sdB candidates.

\begin{tabular}{lcclr}
\hline \hline Star & $\begin{array}{c}\text { Telescope/ } \\
\text { Instrument }\end{array}$ & Date & $R$ & $S / N$ \\
\hline PG 0229+064 & WHT/ISIS & 19981004 & 5700 & 100 \\
PG 0240+046 & WHT/ISIS & 19981004 & 5700 & 150 \\
PG 0902+057* & WHT/UES & 19950506 & $2500^{\#}$ & 30 \\
HS 1000+471 & WHT/ISIS & 19960531 & 2900 & 20 \\
PG 1127+019 & INT/IDS & 20020428 & 4500 & 60 \\
TON 107 & WHT/ISIS & 19960531 & 2900 & 40 \\
PG 1415+492 & INT/IDS & 20020429 & 4500 & 50 \\
PG 1544+488* & INT/IDS & 20020430 & 4500 & 40 \\
PG 1554+408* & WHT/ISIS & 19960531 & 2900 & 60 \\
PG 1600+171* & WHT/ISIS & 19960531 & 2900 & 30 \\
PG 1615+413* & WHT/ISIS & 19960531 & 2900 & 40 \\
PG 1658+273 & WHT/ISIS & 19960531 & 2900 & 70 \\
PG 1715+273 & WHT/ISIS & 19960531 & 2900 & 35 \\
HS 1844+637 & WHT/ISIS & 19960531 & 2900 & 15 \\
LS IV-14 116 & WHT/ISIS & 19981004 & 5700 & 100 \\
PG 2258+155 & WHT/ISIS & 19981004 & 5700 & 70 \\
PG 2321+214* & WHT/ISIS & 19981004 & 5700 & 100 \\
\hline
\end{tabular}

* Stars referred to with different identifier elsewhere.

\# The original UES resolution was $\sim 45000$.

a three-dimensional rectangular grid defined by $T_{\text {eff }}=$ $15000(5000) 40000 \mathrm{~K}, \log g=4.0(0.5) 6.0$ and $n_{\mathrm{He}}=$ $0.1000,0.5000$ and 0.8990 .

The synthetic high resolution spectra were calculated on a wavelength interval of 3900-5000 . Owing to the moderate resolution of the observed spectra, we could not solve independently for microturbulent velocity, rotational velocity or elemental abundances other than hydrogen and helium. Thus, $v_{\mathrm{t}}=5.0 \mathrm{~km} \mathrm{~s}^{-1}, v_{\text {rot }} \sin i=0 \mathrm{~km} \mathrm{~s}^{-1}$ and solar metal abundances were assumed.

Whilst it is possible to compute model atmospheres and synthetic spectra in the LTE approximation for $T_{\text {eff }}>40000 \mathrm{~K}$, departures from LTE at these temperatures make such models increasingly unrealistic. Therefore, where our analyses indicate a star is hotter than this limit, we have chosen not to publish measurements of $T_{\mathrm{eff}}, \log g$ and $n_{\mathrm{He}}$.

\subsection{SFIT2}

The data were analysed using the most recent version of the code SFIT (Jeffery et al. 2001a) which obtains physical parameters, effective temperature ( $\left.T_{\text {eff }}\right)$, surface gravity $(\log g)$ and helium abundance $\left(n_{\mathrm{He}}\right)$ by finding the best-fit solution within a grid of theoretical models by $\chi^{2}$ minimization (Jeffery \& Aznar Cuadrado 2001b). SFIT2 is a rationalisation that combines SFIT and SFIT_SYNTH into a single code capable of modelling both single and binary star spectra.

\subsection{Results}

The blue spectra of He-sdB candidates are shown in Fig. 1. Results from the sFIT2 analysis of the He-sdB stars are presented in Table 2.

Figure 2 shows an expanded region of the spectra dominated by HeI and HeIr absorption lines and the corresponding 


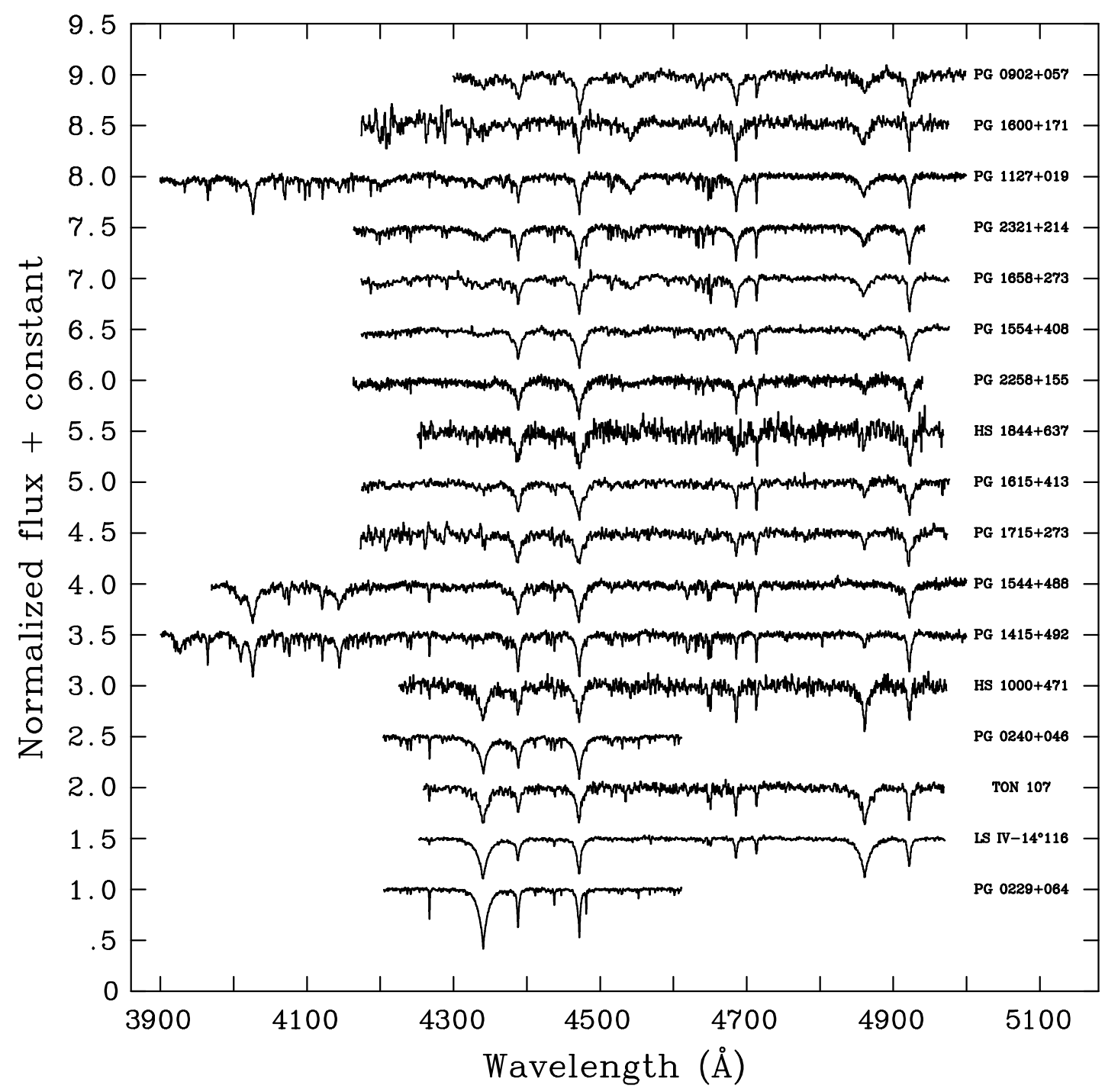

Fig. 1. Blue spectra of He-sdB candidates.

best fitting model. It is clear that some of the stars earlier classified as He-sdB stars (e.g. Jeffery et al. 1996), while having relatively high $\mathrm{He} / \mathrm{H}$ ratios, are not extremely helium-rich.

PG 1600+171 and PG 0902+057 have not been analysed since their $T_{\text {eff }}>40000 \mathrm{~K}$.

\subsection{Errors}

The formal errors associated with the best fit parameters produced by SFIT 2 are unrealistically small. To get a realistic estimate of the random errors in the $\chi^{2}$ fitting procedure in SFIT2 we carried out tests using the high resolution model spectra. We took a high resolution model spectrum and binned it to match the resolution of our observed spectra. The binned spectrum was then smoothed using a Gaussian function to simulate the instrumental broadening. This formed our test model spectrum. Random Gaussian noise representing a given $S / N$ was added to our test model spectrum to get a "realistic" test spectrum. This test spectrum was then analysed with sFIT 2 with $T_{\text {eff }}, \log g$ and $n_{\mathrm{He}}$ all allowed to vary at the same time. This was done a number of times and for several $S / N$ levels. This enabled us to realistically estimate the random errors on the various parameters which are dominant in our fitting procedure. The variance of each parameter obtained from each series of tests gives the random error at a given $S / N$. The estimated random errors range from $\pm 650-150 \mathrm{~K}$ for $T_{\text {eff }}$ and $\pm 0.2-0.1$ dex for $\log g$ corresponding to $S / N$ ranging from $15-150$. The random errors in helium abundance $\left(n_{\mathrm{He}}\right)$ are estimated to be \pm 0.01 .

There are systematic errors associated with the procedures followed in creating the test spectrum and the continuum renormalization procedure in sFrT2 (Jeffery et al. 2001a). We are unable to realistically estimate these as well as those associated with the model atmospheres. The systematic errors are significant but since they cannot be realistically estimated, the errors quoted should be treated as the lower limit of the total error.

\subsection{Previous measurements and references}

A few He-sdB stars have been included in previous spectroscopic studies. Hence it is possible to compare our results and 


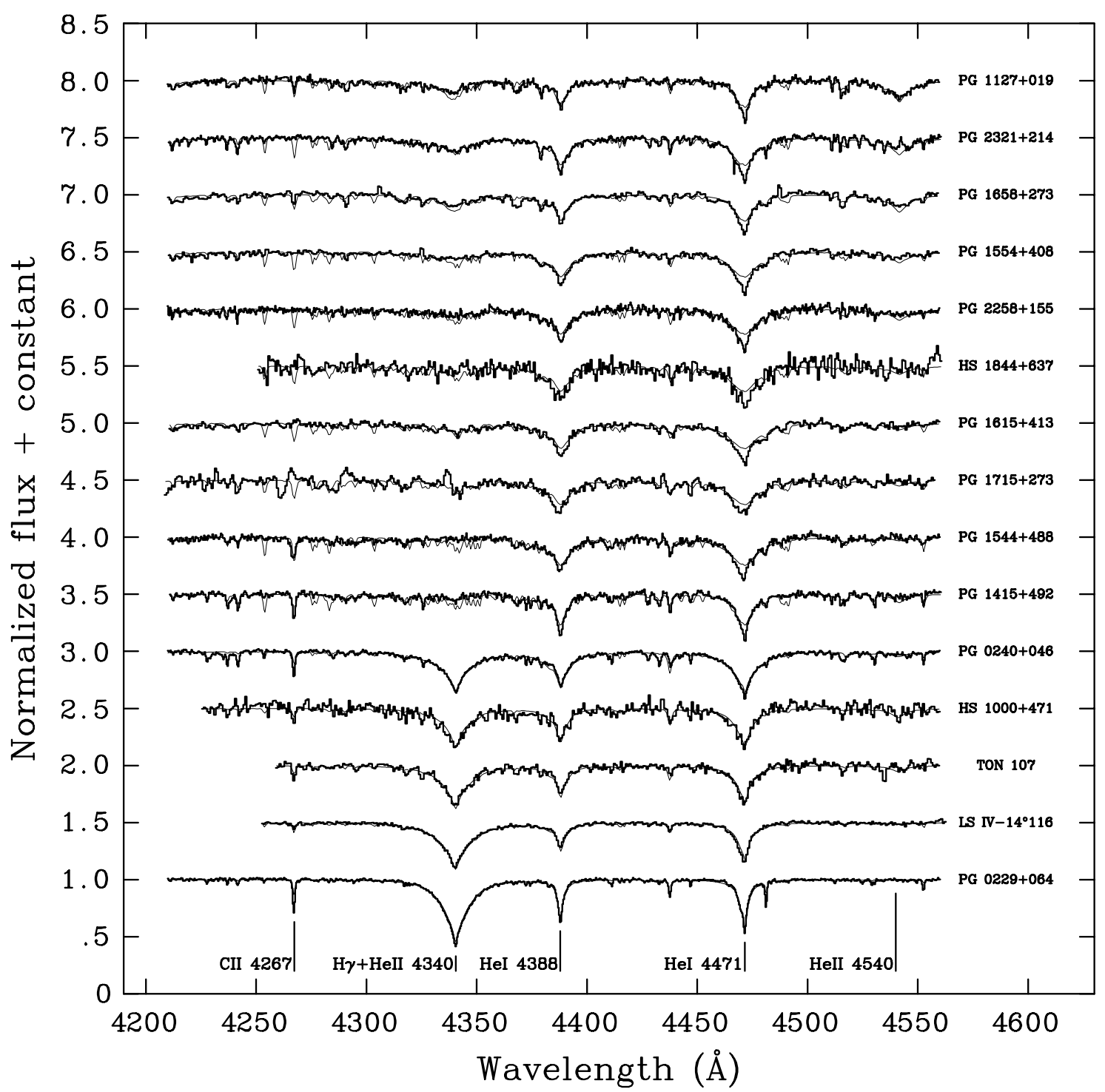

Fig. 2. Blue spectra of He-sdB candidates (thick line) along with the best fitting model spectra obtained using sFIT2.

check for consistency. We compare our results with previous measurements in Table 3 and discuss some of the stars in this section.

PG $0229+064$ was classified a He-sdB star by Aznar Cuadrado \& Jeffery (2002b) because its helium abundance was above average for typical sdB stars. However, with $n_{\mathrm{He}}=0.18$, and a Drilling helium subtype He13, this does not fit our current criteria. Our estimated $T_{\text {eff }}$ and $\log g$ match previous measurements quite well. Saffer et al. (1997) assumed a normal composition in their analysis which probably led to their overestimate of the gravity.

PG 0240+046 was classified He-sdO by Thejll et al. (1994) and He-sdB by Aznar Cuadrado \& Jeffery (2002b). With its high helium abundance $\left(n_{\mathrm{He}}=0.63\right)$, this star meets our current criterion for a $\mathrm{He}-\mathrm{sdB}$ star $(\mathrm{He} / \mathrm{H}$ index 24$)$. We see a significant difference in $\log g$ compared to that obtained by Aznar Cuadrado \& Jeffery (2002b). This can be attributed to the model grid they used which had a helium abundance upper limit, lower than that measured in PG $0240+046$. Our results match reasonably well with those obtained using NLTE models by Thejll et al. (1994).

PG 0902+057 was analysed using NLTE models and classified He-sdO by Thejll et al. (1994). The $T_{\text {eff }}$ for this star is greater than $40000 \mathrm{~K}$ and hence we cannot analyse this star reliably.

\subsection{LTE vs. NLTE}

In Fig. 2, we can see that the models do not fit the cores of some of the HeI lines of the observed spectra, this might be due to NLTE effects which we have not considered. We see that the cores of Her lines of one of our targets analysed with NLTE models (Thejll et al. 1994) fit much better than our LTE models although the $T_{\text {eff }}$ and $\log g$ match quite well. On the other hand Moehler et al. (1997), despite using NLTE models, were not able to fit the cores of some of the Her lines in the HesdB star F2-2. This problem is well known for more luminous helium stars (Jeffery \& Heber 1992) and still awaits a solution. 


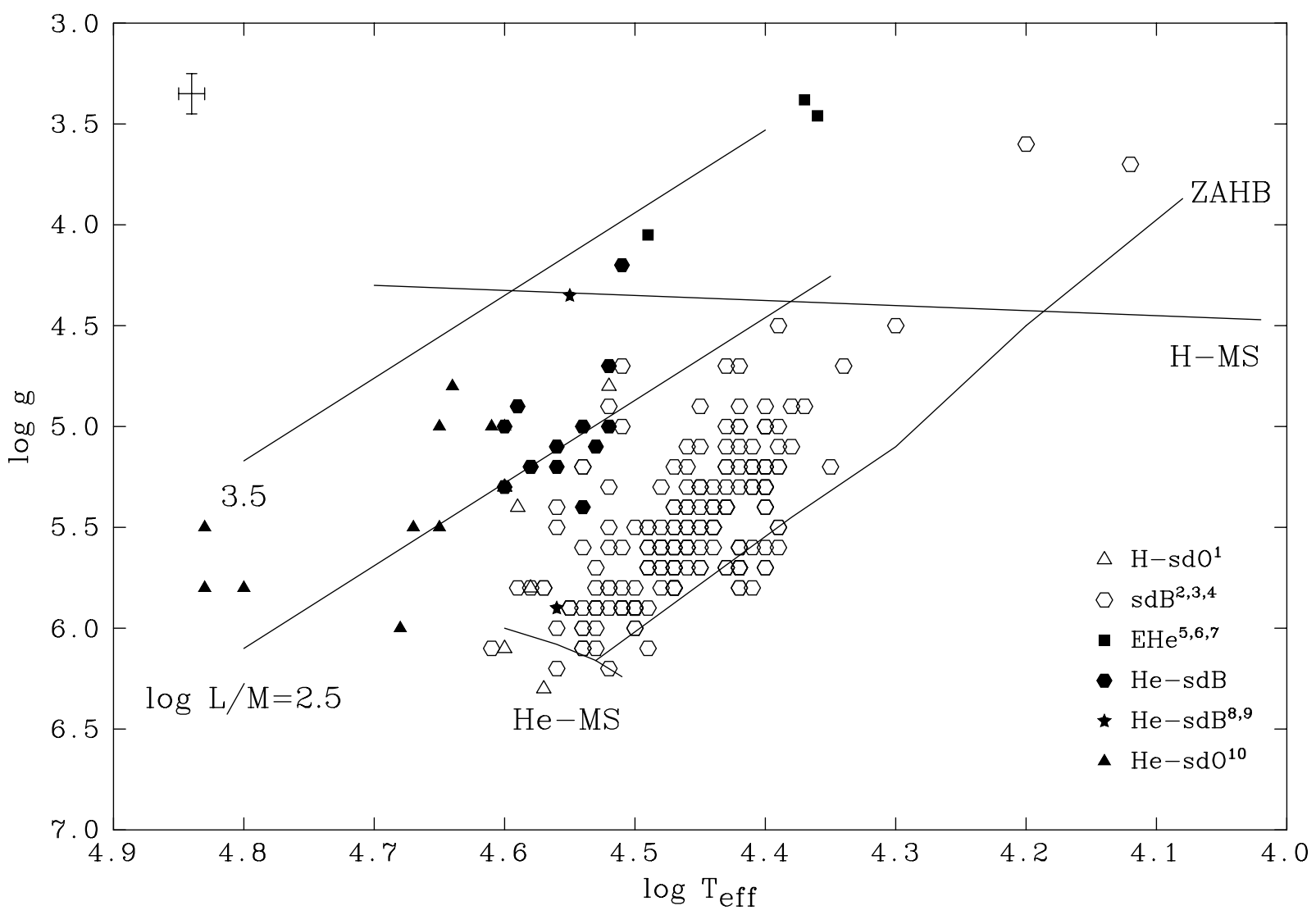

Fig. 3. Position of He-sdB stars on the $\log g-T_{\text {eff }}$ diagram in comparison to some other subluminous stars. The error bar corresponds to the errors at $S / N=70$.

References: ${ }^{1}$ Moehler et al. (1990a); ${ }^{2}$ Moehler et al. (1990b); ${ }^{3}$ Saffer et al. (1994); ${ }^{4}$ Maxted et al. (2001); ${ }^{5}$ Jeffery et al. (2001a); ${ }^{6}$ Woolf \& Jeffery (2000); ${ }^{7}$ Jeffery (1998); ${ }^{8}$ Moehler et al. (1997); ${ }^{9}$ Moehler et al. (2002); ${ }^{10}$ Dreizler et al. (1990).

Table 2. Physical parameters for He-sdB stars.

\begin{tabular}{ccccccc}
\hline \hline Star & $T_{\text {eff }}[\mathrm{K}]$ & $\log g[\mathrm{cgs}]$ & $n_{\text {He }}$ & Class & Ref. & Class (4) \\
\hline PG 0229+064 & $19700 \pm 150$ & $4.2 \pm 0.1$ & $0.18 \pm 0.01$ & sdOA & 1 & sdB3V:He13 \\
PG 0240+046 & $34000 \pm 150$ & $5.4 \pm 0.1$ & $0.63 \pm 0.01$ & sdOB & 1 & sdBC0.2VII:He24 \\
PG 0902+057 & $>40000$ & & & sdOD & 1 & sdB0VII:He38 \\
HS 1000+471 & $33300 \pm 550$ & $4.7 \pm 0.2$ & $0.58 \pm 0.01$ & He-sdB & 2 & sdBC0.2VII:He28 \\
PG 1127+019 & $39900 \pm 200$ & $5.0 \pm 0.1$ & $0.99 \pm 0.01$ & sdOD & 1 & sdOC9VII:He40 \\
TON 107 & $33300 \pm 300$ & $5.0 \pm 0.1$ & $0.36 \pm 0.01$ & sdOD & 1 & sdBC0.5VII:He28 \\
PG 1415+492 & $32200 \pm 250$ & $4.2 \pm 0.1$ & $0.99 \pm 0.01$ & sdOD & 1 & sdBC1VI:He39 \\
PG 1544+488 & $34000 \pm 300$ & $5.1 \pm 0.1$ & $0.99 \pm 0.01$ & sdOD & 1 & sdBC1VIII:He39 \\
PG 1554+408 & $37700 \pm 200$ & $5.2 \pm 0.1$ & $0.99 \pm 0.01$ & sdOD & 1 & sdB0.2VII:He39 \\
PG 1600+171 & $>40000$ & & & sdOD & 1 & sdOC8.5VII:He39 \\
PG 1615+413 & $36000 \pm 300$ & $5.2 \pm 0.1$ & $0.99 \pm 0.01$ & sdOD & 1 & sdB1VII:He37 \\
PG 1658+273 & $38800 \pm 200$ & $4.9 \pm 0.1$ & $0.99 \pm 0.01$ & sdOD & 1 & sdOC9.5VII:He39 \\
PG 1715+273 & $34700 \pm 300$ & $5.0 \pm 0.1$ & $0.99 \pm 0.01$ & sdOD & 1 & sdB1VII:He37 \\
HS 1844+637 & $36400 \pm 650$ & $5.1 \pm 0.2$ & $0.99 \pm 0.01$ & He-sdB & 2 & sdB1VII:He39 \\
LS IV-14 116 & $32500 \pm 150$ & $5.4 \pm 0.1$ & $0.21 \pm 0.01$ & He-sdO & 3 & sdB0.2VII:He17 \\
PG 2258+155 & $37700 \pm 200$ & $5.2 \pm 0.1$ & $0.99 \pm 0.01$ & sdOD & 1 & sdB0.2VII:He39 \\
PG 2321+214 & $39600 \pm 150$ & $5.3 \pm 0.1$ & $0.99 \pm 0.01$ & sdOD & 1 & sdB0VII:He37 \\
\hline
\end{tabular}

References: 1: Green et al. (1986); 2: Heber et al. (1996); 3: Viton et al. (1991); 4: Drilling et al. (2003). 


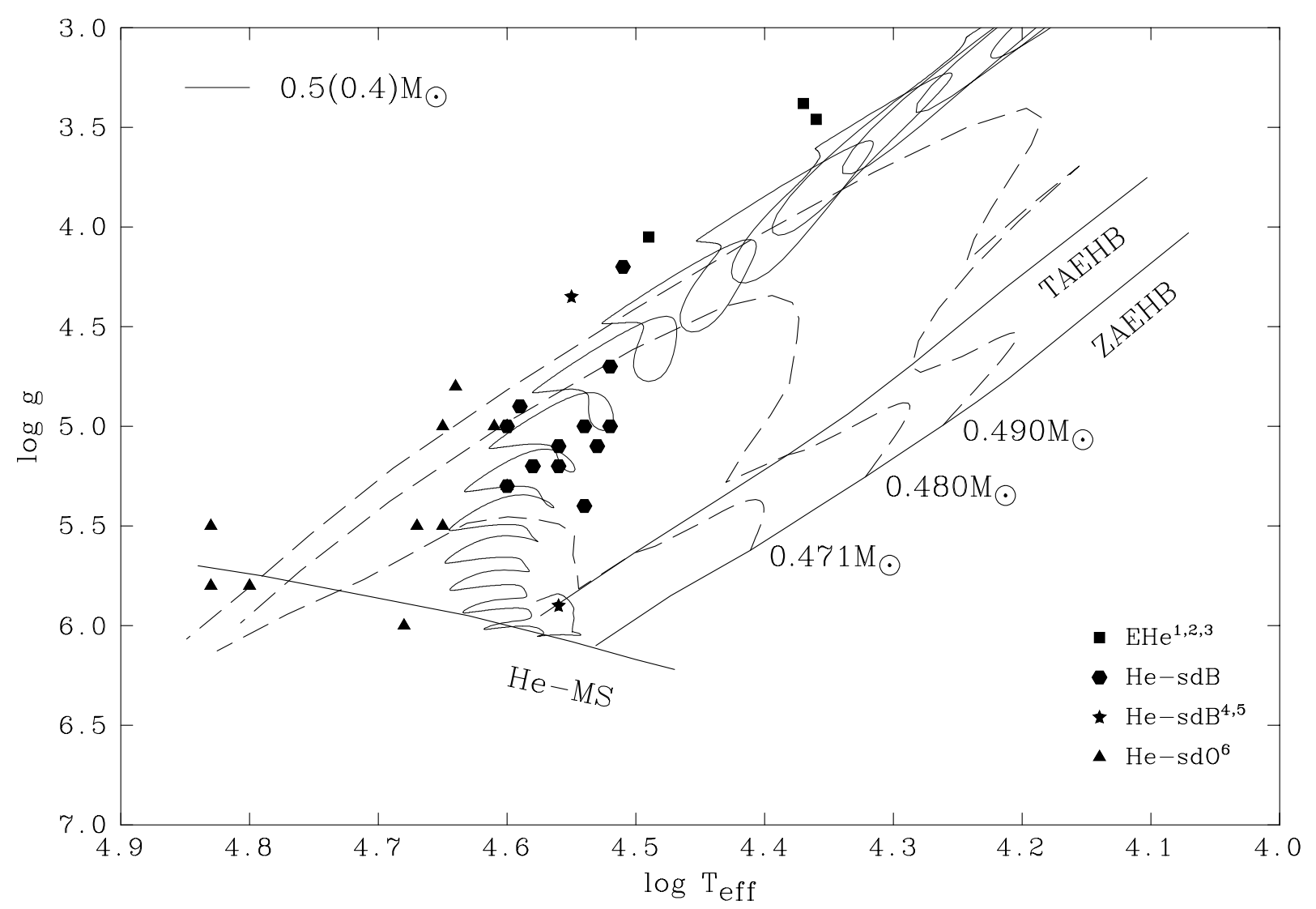

Fig. 4. Helium-rich stars on the $\log g-T_{\text {eff }}$ diagram plotted with evolutionary tracks. Single star tracks (dashed lines) are taken from Dorman et al. (1993) and merged He+He WD tracks (solid lines) are taken from Saio \& Jeffery (2000).

References: ${ }^{1}$ Jeffery et al. (2001a); ${ }^{2}$ Woolf \& Jeffery (2000); ${ }^{3}$ Jeffery (1998); ${ }^{4}$ Moehler et al. (1997); ${ }^{5}$ Moehler et al. (2002); ${ }^{6}$ Dreizler et al. (1990).

Kudritzki (1979) has shown that the NLTE effect can be ignored for $T_{\text {eff }}<35000 \mathrm{~K}$ in hot stars. With the quality of our data (low $S / N$ and moderate resolution), NLTE analysis would not contribute to any significant difference to the values obtained with our LTE analysis.

\section{Discussion}

The evolution of sdB stars is still under debate. A number of different binary star scenarios would seem to account for the majority (Green et al. 2001; Maxted et al. 2002; Aznar Cuadrado \& Jeffery 2002b). In addition, single star evolution with enhanced mass-loss (D'Cruz et al. 1996) might account for the remainder of apparently single sdB stars, and the extreme horizontal branch (EHB) stars in globular clusters, whilst some fraction may be produced by the merger of two helium white dwarfs (WD) (Iben 1990; Saio \& Jeffery 2000).

Our analysis of He-sdB stars addresses directly the question of whether these stars are related to the "normal" sdB stars. If so, are they formed by the same process with two different outcomes, or does an He-sdB become an sdB or vice versa? If not, how otherwise are He-sdB stars formed? With substantial recent progress in understanding the origin of normal sdB stars, the He-sdB stars represent a persistent challenge.

The location of the He-sdB stars is compared with that of other low-mass stellar remnants in the $\log g-T_{\text {eff }}$ diagram (Fig. 3). We see that the He-sdB stars are systematically more luminous than hydrogen-rich sdB stars because, for the same $T_{\text {eff }}$, their gravities are lower by $\sim 1$ dex.

On the other hand, He-sdB stars lie between the highgravity helium-rich sdO (He-sdO) stars (Dreizler et al. 1990) and the highest gravity extreme helium (EHe) stars, V652 Her (Jeffery et al. 2001a), BX Cir (Woolf \& Jeffery 2000) and LS IV $+6^{\circ} 2$ (Jeffery 1998). This leads the authors to suspect an evolutionary connection between some or all of these stars.

Figure 4 compares the position of hydrogen-poor remnants with evolution tracks for EHB stars (Dorman et al. 1993) and for $\mathrm{He}+\mathrm{He}$ WD mergers (Saio \& Jeffery 2000). The He-sdB stars are connected to sdB stars by the post-EHB evolution tracks, but these cannot explain the surface abundances. The $\mathrm{He}+\mathrm{He}$ WD merger tracks connect EHe stars, He-sdB stars and He-sdO stars very well, but predict helium-rich CNOprocessed surfaces. So far, only V652 Her is known to have a purely $\mathrm{CNO}$-processed surface and be a likely $\mathrm{He}+\mathrm{He} \mathrm{WD}$ merger.

Brown et al. (2001) have argued that EHB stars in globular clusters have evolved from red giant stars with high mass loss that undergo a late helium-core flash on the WD cooling curve. The core flash leads to convective-flash mixing of the envelope. They further suggest that such flash-mixing provides a possible explanation for $\mathrm{He}-\mathrm{sdB}$ and $\mathrm{He}-\mathrm{sdO}$ stars, which would lend support to the late core-flash hypothesis. However, our analyses 
Table 3. Comparison of our results with the ones in the literature.

\begin{tabular}{|c|c|c|c|c|}
\hline Star & $T_{\text {eff }}[\mathrm{K}]$ & $\log g$ [cgs] & $n_{\mathrm{He}}$ & Reference \\
\hline \multirow[t]{8}{*}{ PG 0229+064 } & $19700 \pm 150$ & $4.20 \pm 0.10$ & $0.18 \pm 0.01$ & SFIT2 \\
\hline & $18000 \pm 1025$ & $4.35 \pm 0.10$ & $0.25 \pm 0.01$ & Aznar Cuadrado \& Jeffery (2002b) \\
\hline & $20100 \pm 400$ & & & Aznar Cuadrado \& Jeffery (2002a) \\
\hline & $19000 \pm 950$ & $4.55 \pm 0.10$ & 0.14 & Ramspeck et al. (2001) \\
\hline & $20353 \pm 200$ & $4.82 \pm 0.05$ & & Saffer et al. (1997) \\
\hline & $22000 \pm 1000$ & $4.65 \pm 0.30$ & $0.12 \pm 0.01$ & Saffer et al. (1994) \\
\hline & 20200 & & & Ramspeck et al. $(2001)^{1}$ \\
\hline & 21000 & & & Ramspeck et al. $(2001)^{2}$ \\
\hline \multirow[t]{4}{*}{ PG $0240+046$} & $34000 \pm 150$ & $5.40 \pm 0.10$ & $0.63 \pm 0.01$ & SFIT2 \\
\hline & $36200 \pm 400$ & $6.25 \pm 0.10$ & $0.66 \pm 0.02$ & Aznar Cuadrado \& Jeffery (2002b) \\
\hline & $34800 \pm 1850$ & & & Aznar Cuadrado \& Jeffery (2002a) \\
\hline & $37000 \pm 2000$ & $5.30 \pm 0.30$ & $0.55 \pm 0.11$ & Thejll et al. (1994) \\
\hline \multirow[t]{2}{*}{ PG $0902+057$} & $>40000$ & & & SFIT2 \\
\hline & $44000 \pm 2000$ & $6.00 \pm 0.10$ & $0.97 \pm 0.19$ & Thejll et al. (1994) \\
\hline \multirow[t]{2}{*}{ PG $1544+488$} & $34000 \pm 300$ & $5.10 \pm 0.10$ & $0.99 \pm 0.01$ & SFIT2 \\
\hline & 31000 & 5.10 & $\leq 0.99$ & Heber et al. (1988) \\
\hline \multirow[t]{3}{*}{ LS IV-14 116} & $32500 \pm 150$ & $5.40 \pm 0.10$ & $0.21 \pm 0.01$ & SFIT2 \\
\hline & 35000 & & & Ulla \& Thejll (1998) \\
\hline & $33000 \pm 1000$ & $5.80 \pm 0.20$ & $0.20 \pm 0.07$ & Viton et al. (1991) \\
\hline \multirow[t]{2}{*}{ PG $2321+214$} & $39600 \pm 150$ & $5.30 \pm 0.10$ & $0.99 \pm 0.01$ & SFIT2 \\
\hline & 43000 & & & Thejll et al. ${ }^{3}$ \\
\hline
\end{tabular}

Temperature calculated from photometry : ${ }^{1}$ Wesemael et al. (1992); ${ }^{2}$ Moehler et al. (1990b).

${ }^{3}$ Thejll et al. (unpublished) reported by Ulla \& Thejll (1998).

of He-sdB stars show that these, at least, do not lie on any of the evolutionary sequences presented by these authors. On the contrary, they are too luminous by $\sim 1$ dex to be flash-mixed EHB stars.

A crucial question therefore concerns the relative abundances of carbon, nitrogen and oxygen in the He-sdB stars. It may be seen (Fig. 2) that while several He-sdB stars show a strong CII $\lambda 4267$ line, a few show none (the models all assume a solar carbon abundance). Strong carbon would preclude an $\mathrm{He}+\mathrm{He}$ WD merger origin for these stars; some other mechanism, possibly a $\mathrm{He}+\mathrm{CO}$ WD merger (Saio \& Jeffery 2002), may be responsible. Detailed abundance analyses using highresolution spectra will be necessary to address this question.

It is very interesting that helium-rich "sdB" stars have also been observed in globular clusters M15 (Moehler et al. 1997) and $\omega$ Cen (Moehler et al. 2002). Whilst the former (F2-2 in M15) seems to have a much higher gravity than our He-sdB stars, the $S / N$ of the data is low. The star D10763 in $\omega$ Cen, on the other hand, lies much closer to the He-sdB stars in our current sample, in the $\log g-T_{\text {eff }}$ diagram (Figs. 3 and 4). The dilemma, for D10763, is that with $V=19.1$, its surface gravity suggests a distance of $50 \mathrm{kpc}$ were it to have a mass of $0.5 M_{\odot}$ (Moehler et al. 2002). From our derived gravities and assuming a mass of $0.5 M_{\odot}$, we estimate our He-sdB candidates, with $m_{\mathrm{v}}$ in the range $12.8-17.1 \mathrm{mag}$, to lie at distances ranging from $2.1-15.1 \mathrm{kpc}$.

\section{Conclusion}

We have analysed a sample of stars with spectra dominated by neutral helium and with high surface gravities and generically known as helium-rich subdwarf B stars. Their effective temperatures, surface gravities and helium abundances have been derived from medium resolution blue spectra and LTE models atmospheres. We find that the He-sdB stars have systematically lower surface gravities than normal hydrogen-rich subdwarf B stars and may form part of a sequence connecting high-gravity extreme helium stars and helium-rich subdwarf $\mathrm{O}$ stars.

Various evolutionary scenarios have been considered to explain the He-sdB stars, including post horizontal-branch evolution, the merger of two helium white dwarfs, and the late hot flasher scenario for post giant-branch evolution. More detailed quantitative abundance analyses will be required to resolve which of these, if any, are responsible for the production of He-sdB stars.

Acknowledgements. This research is supported by a grant to the Armagh Observatory from the Northern Ireland Department of Culture, Arts and Leisure and by the UK Particle Physics and Astronomy Research Council (PPARC) through the award of telescope time and travel grants. The authors acknowledge the data analysis facilities provided by the Starlink Project which is run by CCLRC on behalf of PPARC. This research has made use of NASA's Astrophysics Data System. This research has also made use of the SIMBAD database, operated at CDS, Strasbourg, France. 


\section{References}

Aznar Cuadrado, R., \& Jeffery, C. S. 2002a, A\&A, 368, 994

Aznar Cuadrado, R., \& Jeffery, C. S. 2002b, A\&A, 385, 131

Brown, T. M., Ferguson, H. C., Davidsen, A. F., \& Dorman, B. 1997 , ApJ, 482, 685

Brown, T. M., Sweigart, A. V., Lanz, T., Landsman, W. B., \& Hubeny, I. 2001, ApJ, 562, 368

D’Cruz, N. L., Dorman, B., Rood, R. T., \& O'Connell, R. W. 1996, ApJ, 466, 359

Dorman, B., Rood, R. T., \& O’Connell, R. W. 1993, ApJ, 419, 596

Dreizler, S., Heber, U., Werner, K., Moehler, S., \& de Boer, K. S. 1990, A\&A, 235, 234

Drilling, J. S. 1996, in Hydrogen Deficient Stars, ed. C. S. Jeffery, \& U. Heber, ASP Conf. Ser., 96, 461

Drilling, J. S., Jeffery, C. S., Moehler, S., Heber, U., \& Napiwotzki, R. 2003, in preparation

Green, R. F., Schmidt, M., \& Liebert, J. 1986, ApJS, 61, 304

Green, E. M., Liebert, J., \& Saffer, R. A. 2001, 12th European Workshop on White Dwarfs, ASP Conf. Ser., 226, 192

Heber, U. 1986, A\&A, 155, 33

Heber, U., Dreizler, S., de Boer, K. S., Moehler, S., \& Richtler, T. 1988, Astron. Gesellschaft Abstract Series, 1, 16

Heber, U., Dreizler, S., Werner, K., Engels, D., \& Hagen, H.-J. 1996, in Hydrogen Deficient Stars, ed. C. S. Jeffery, \& U. Heber, ASP Conf. Ser., 96, 241

Heber, U., Edelmann, H., Lemke, M., Napiwotzki, R., \& Engels, D. 1999, 11th Workshop on White Dwarfs, ed. J.-E. Solheim, \& E. G. Meistas, ASP Conf. Ser., 169, 551

Iben, I. Jr. 1990, ApJ, 353, 215

Jeffery, C. S., \& Heber, U. 1992, A\&A, 260, 133

Jeffery, C. S., Heber, U., Hill, P. W., et al. 1996, in Hydrogen Deficient Stars, ed. C. S. Jeffery, \& U. Heber, ASP Conf. Ser., 96, 471
Jeffery, C. S., Harrison, P. M., \& Drilling, J. S. 1997, A\&A, 125, 501 Jeffery, C. S. 1998, MNRAS, 294, 391

Jeffery, C. S., Woolf, V. M., \& Pollacco, D. L. 2001a, A\&A, 376, 497

Jeffery, C. S., \& Aznar Cuadrado, R. 2001b, 378, 936

Kudritzki, R. P. 1979, The elements and their isotopes in the universe, 22nd Liège International Symp., 295

Maxted, P. F. L., Heber, U., Marsh, T. R., \& North, R. C. 2001, MNRAS, 326, 1391

Maxted, P. F. L., Marsh, T. R., \& Moran, C. K. J. 2002, MNRAS, 332, 745

Moehler, S., de Boer, K. S., \& Heber, U. 1990a, A\&A, 239, 265

Moehler, S., Richtler, T., de Boer, K. S., Dettmar, R. J., \& Heber, U. 1990b, A\&A, 86, 53

Moehler, S., Heber, U., \& Durrell, P. R. 1997, A\&A, 317, L83

Moehler, S., Sweigart, A. V., Landsman, W. B., \& Dreizler, S. 2002, A\&A, 395, 37

Ramspeck, M., Heber, U., \& Edelmann, H. 2001, A\&A, 378, 907

Saffer, R. A., Bergeron, P., Koester, D., \& Liebert, J. 1994, ApJ, 432, 351

Saffer, R. A., Keenman, F. P., Hambly, N. C., Dufton, P. L., \& Liebert, J. 1997, ApJ, 491, 172

Schulz, H., Heber, U., \& Wegner, G. 1991, PASP, 103, 435

Saio, H., \& Jeffery, C. S. 2000, MNRAS, 313, 671

Saio, H., \& Jeffery, C. S. 2002, MNRAS, 333, 121

Thejll, P., Bauer, F., Saffer, R., et al. 1994, ApJ, 433, 819

Ulla, A., \& Thejll, P. 1998, A\&AS, 132, 1

Viton, M., Deleuil, M., Tobin, W., Prévot, L., \& Bouchet, P. 1991, A\&A, 242, 175

Wesemael, F., Fontaine, G., Bergeron, P., Lamontagne, R., \& Green, F. F. 1992, AJ, 104, 203

Woolf, V. M., \& Jeffery, C. S. 2000, MNRAS, 318, 974 